\title{
Energetic ion observations in the middle Jovian magnetosphere
}

\author{
C. M. S. Cohen and E. C. Stone \\ George W. Downs Laboratory of Physics, California Institute of Technology, Pasadena, California, USA
}

\author{
R. S. Selesnick \\ The Aerospace Corporation, Los Angeles, California, USA
}

\begin{abstract}
The middle magnetosphere of Jupiter has not been well studied prior to the Galileo mission. This region (10-30 $R_{J}$ ) has been traversed by Galileo numerous times allowing spatial and temporal features to be differentiated. We present observations of energetic ions $(E \sim 4.5-$ 18.4 $\mathrm{MeV}_{\text {nucleon }}{ }^{-1}$ ) in the middle Jovian magnetosphere obtained with the Heavy Ion Counter (HIC) on Galileo. The radial dependence of the intensity, spectrum, and composition of these particles indicates that solar material dominates the 15 to $30 R_{J}$ region and diffuses without loss toward Jupiter. Near $10 R_{J}$ the composition is more Iogenic and shows evidence of strong losses. These observations can be understood in terms of a common energy per charge spectrum for all heavy ions. Due to the substantially different charge to mass ratios of the logenic and solar ions, the $\mathrm{O} / \mathrm{C}$ and $\mathrm{S} / \mathrm{C}$ abundance ratios vary with magnetic moment and therefore radial distance when observed over the fixed energy interval of the HIC instrument. Near $25 R_{J}$ there is evidence of acceleration of ions to $\mathrm{MeV}$ nucleon $^{-1}$ energies. The location of this acceleration has been suggested previously but not directly observed before the Galileo mission.
\end{abstract}

\section{Introduction}

Prior to the Galileo mission, five spacecraft had flown through the Jovian magnetosphere. During these passes energetic particle data from tens of $\mathrm{keV}$ nucleon ${ }^{-1}$ to tens of $\mathrm{MeV}$ nucleon ${ }^{-1}$ energies were obtained over various regions of the magnetosphere. Overviews of the observations and conclusions resulting from the Pioneer 10,11 and Voyager 1, 2 encounters are available from Krimigis and Roelof [1983] and Schardt and Goertz [1983]. Results from the Ulysses encounter [e.g., Lanzerotti et al., 1992; Simpson et al., 1992, and references therein] furthered the understanding of particle dynamics and composition but also yielded results that were significantly different from those obtained by the previous spacecraft encounters, indicating that the Jovian magnetosphere was an extremely dynamic system. Data from the Energetic Particles Detector (EPD [Williams et al., 1992]) and the Heavy Ion Counter (HIC [Garrard et al., 1992]) on Galileo provide the opportunity to distinguish spatial and temporal characteristics of energetic particles.

In this paper we focus on ions with energies above a few $\mathrm{MeV}$ nucleon $^{-1}$. In this energy regime the energetic particle environment of the inner Jovian magnetosphere has been well studied by instruments on the Voyager 1, 2 [Vogt et al., 1979a, 1979b; Gehrels et al., 1981; Gehrels and Stone, 1983], Pioneer 10, 11 [Trainor et al., 1974], Ulysses [Simpson et al., 1992], and Galileo [Garrard et al., 1996; Cohen et al., 2000] missions. The heavy ion composition is predominantly oxygen and sulfur with smaller amounts of sodium. The source of this material is the volcanic moon Io which emits high-velocity neutrals that are ionized, accelerated to energies of tens of $\mathrm{MeV}_{\text {nucleon }}{ }^{-1}$, and

Copyright 2001 by the American Geophysical Union.

Paper number 2001JA000008.

0148-0227/01/2001JA000008\$09.00 diffuse back into the inner magnetosphere as singly charged energetic particles. Typical energy spectra obtained above a few $\mathrm{MeV}$ nucleon ${ }^{-1}$ are steep power laws with indices of -6 to -10 and tend to soften with decreasing distance from Jupiter due to strong pitch angle scattering [Gehrels and Stone, 1983; Cohen et al., 2000].

In contrast, the outer Jovian magnetosphere has not been thoroughly examined above energies of several $\mathrm{MeV}^{\text {nucleon }}{ }^{-1}$. Limited data from Voyager 1, 2 indicate that the composition is similar to that of solar particles [Vogt et al., 1979a, 1979b], suggesting a nonmagnetospheric source which enters the magnetosphere at an undetermined location. No spectra have been obtained in this region.

A transition between the different energetic particle characteristics of the inner and outer magnetosphere occurs somewhere roughly between 10 and $30 R_{J}$, a region we refer to in this paper as the middle magnetosphere. This is a region that has not been well studied and now can be with the data obtained by the Galileo spacecraft. Not only has the middle magnetosphere been traversed multiple times, allowing distinctions to be made between temporal and spatial variations, but the orbits pass through this region over a range of local times. In this work, we use the energetic particle data from the Heavy Ion Counter (HIC) on Galileo obtained during these orbits to investigate the temporal, spectral, spatial, and compositional properties of 4.5 to 18.5 $\mathrm{MeV}$ nucleon ${ }^{-1}$ energetic particles in the middle magnetosphere.

Additionally, the middle magnetosphere may contain the acceleration site of the Iogenic plasma to high energies [Barbosa et al., 1984]. Prior to the Galileo mission, the location of this acceleration region had not been identified, but was suggested to be beyond $15 R$, by Gehrels and Stone [1983] and Barbosa et al. [1984]. Recent energetic particle measurements from HIC have provided evidence of acceleration at 25-30 $R_{J}$ [Selesnick et al., 2001]. More of these data will be presented and discussed here. 

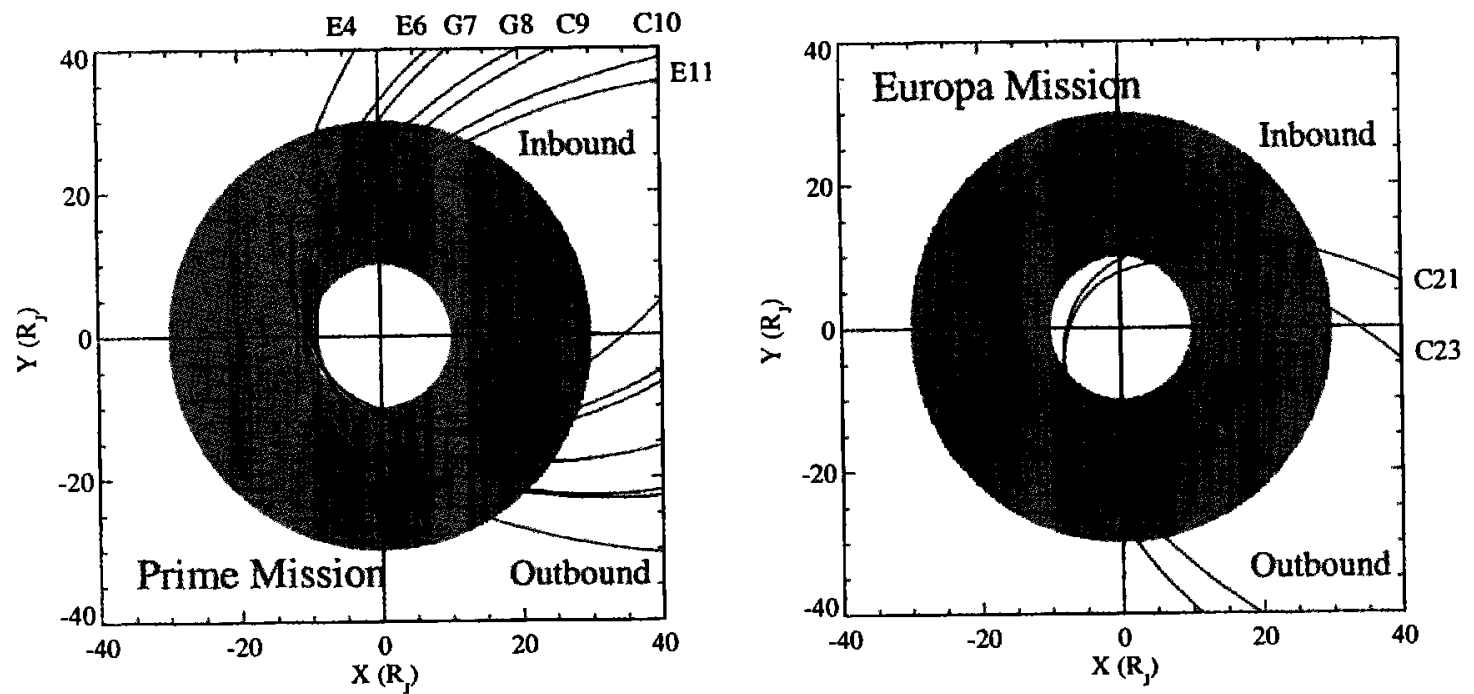

Figure 1. Orbits from the Galileo Prime and Europa missions that are used in this work. The shaded ring denotes the radial region of interest.

\section{Orbit, Instrumentation, and Data Analysis}

Galileo was inserted into Jovian orbit in December 1995. The prime mission consisted of 11 petal-shaped orbits, with apojoves concentrated in the midnight-dawn sector. This mission was followed by the Galileo Europa Mission which consisted of 14 orbits with apojoves primarily in the midnight-dusk sector. Each of these orbits are denoted with a letter indicating the moon targeted for encounter (I for Io, E for Europa, C for Callisto, and $G$ for Ganymede) followed by a number (i for the ith orbit). Thus the fourth orbit, which had Europa as a target, is referred to as E4.

Nine of the 25 orbits contain substantial data in the 10 to $30 R_{J}$ region, E4, E6, G7, G8, C9, C10, E1 1, C21, and C23; see Figure
1. For analysis, each of the orbits was divided into inbound and outbound segments spanning as much of the 10 to $30 R_{J}$ region as the data permitted. Although the inbound segments of C21 and C23 occur closer to midnight local time than the other orbits, in general the orbits allow similar regions of the magnetosphere to be observed several times while also covering a limited range of local times.

The Heavy Ion Counter (HIC) on Galileo measures ions from 4 to $200 \mathrm{MeV}$ nucleon $^{-1}$ and is composed of two telescopes [Garrard et al., 1992]. The telescopes are mounted such that their boresights are nearly perpendicular to the spin axis of the spacecraft, which points toward the Earth. Due to the spinning, the HIC telescopes sample particles at all pitch angles, although the data presented here are of low time resolution such that the

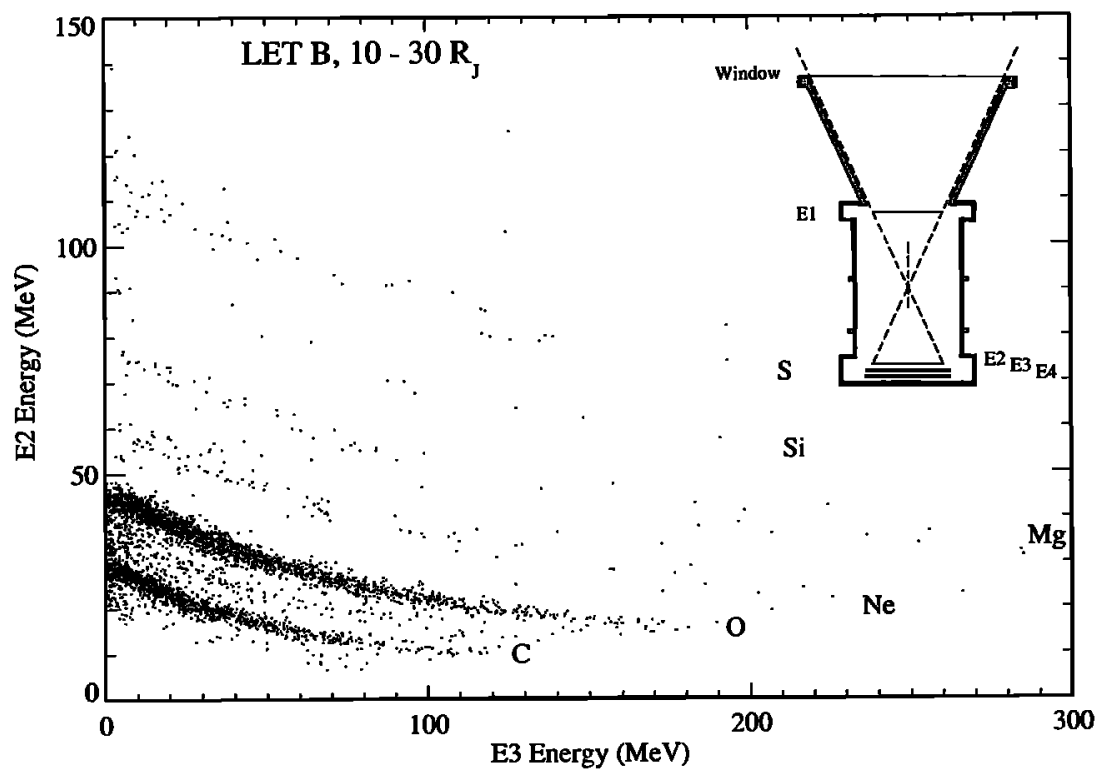

Figure 2. Energies (E2) deposited in the second versus those (E3) measured by the third LET B detector for events stopping in the third LET B detector. Different elements form distinct tracks allowing identification of carbon, oxygen, sulfur, and other ions to be made. The data are from all nine orbits (which occurred between day 3321996 and day 270 1999 ) inbound and outbound from 10 to $30 R$ J. The inset is a schematic of the LET B detector. 


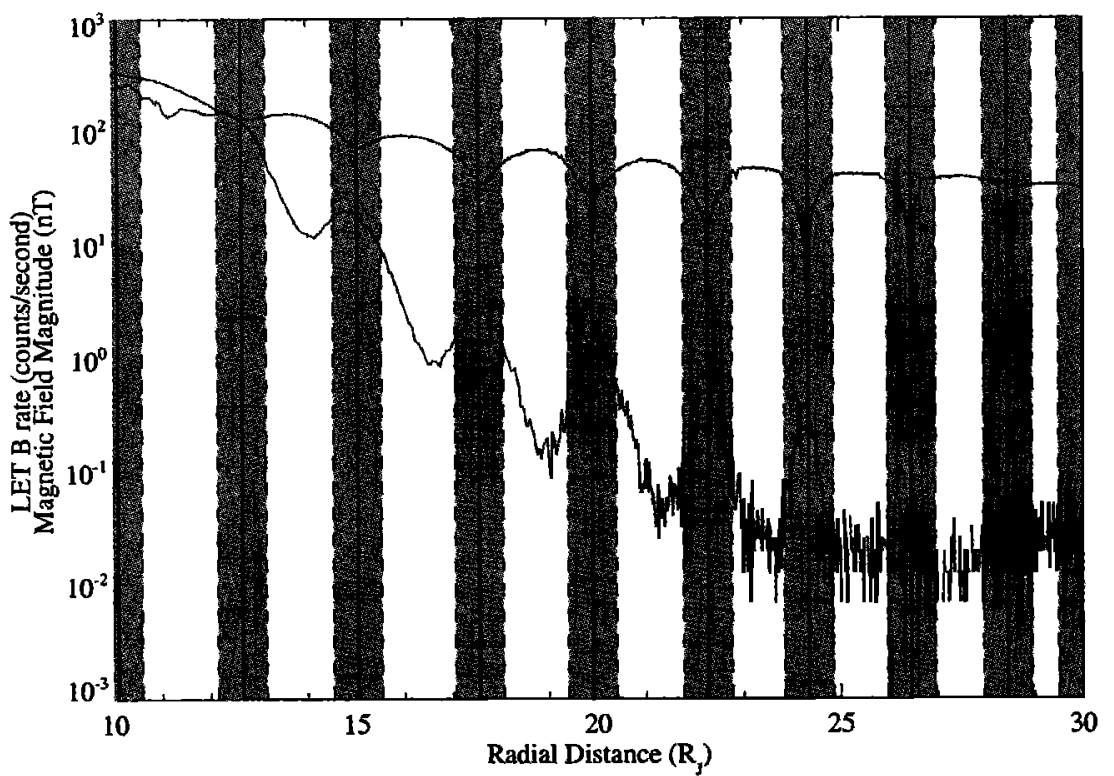

Figure 3. LET B counting rate (bottom trace) and the magnetic field magnitude (top trace; courtesy of the Galileo magnetometer team) versus radial distance from Jupiter for orbit C21 inbound. Shaded regions denote areas used for data analysis (the midpoint of which is based on the local minimum in the magnetic field magnitude which indicates a crossing of the magnetic equator and is indicated by the solid vertical lines).

data from multiple spins are combined and the specific pitch angle distribution cannot be determined.

The LET B telescope, see inset Figure 2, contains four solid state silicon detectors that measure the energy deposited by incoming 4.5 to $18.4 \mathrm{MeV}$ nucleon $^{-1}$ particles. It is clear from Figure 2 that individual elements can be identified when the energy deposited in the second detector (E2) is plotted versus the energy deposited in the third detector (E3) for particles stopping in E3. Similar identification can be made using E1 (the energy deposited in the first detector) and E2 for particles stopping in the second detector. Due to the limited telemetry of the Galileo spacecraft, the energies for only a small fraction of the events are transmitted for this type of analysis. Figure 2 contains the event data for all nine orbits (which occurred between day 3321996 and day 2701999 ) inbound and outbound from 10 to $30 R_{J}$. Rate data are accumulated separately for particles stopping in the second and third detectors and are used to normalize the elemental distributions obtained from the detailed event data in order to obtain an accurate intensity measurement.

For each inbound and outbound segment, LET B event data corresponding to carbon, oxygen, and sulfur nuclei were extracted. Average intensity values were obtained over selected regions of $\pm 0.5 R_{J}$ centered about the position of each magnetic equator crossing as indicated by local minima in the magnetometer data (provided by the Galileo magnetometer team). Figure 3 illustrates this selection process for orbit C21 (shaded regions correspond to selected data). Unfortunately, there is no magnetic field data available for the E6 orbit. It is evident from the peaks in the rate profile of Figure 3 that the heavy ions are primarily contained to the magnetic equator, as was also observed by the Pioneer and Ulysses missions [Trainor et al., 1974; Smith et al., 1992]. Thus for the E6 orbit the magnetic equator crossings were determined from the local maxima in the rate profile. While this is roughly consistent with defining crossings based on magnetometer data, it is less accurate at large radial distances where there are few particles at HIC energies. The selection process was done to avoid having to make intensity corrections that are related to the pitch angle distribution, which is not obtainable from these data, and resulted in typically $-45 \%$ of the available event data between 10 and 30 $R_{J}$ for each orbit being selected. An average radial position was determined for each time period based on the Galileo trajectory. In Plate 1 we present oxygen intensities for each of the nine orbits of interest as a function of radial distance from Jupiter for the inbound and outbound segments.

\section{Radial Variations}

As expected, the oxygen intensity decreases dramatically with increasing distance from Jupiter, dropping $\sim 2$ to 4 orders of magnitude over $10 R_{J}$. Surprisingly, there are significant differences between the inbound and outbound segments. There is substantial variation from orbit to orbit on the inbound segments, while the outbound intensities are more similar for each orbit with the notable exception of E6 which will be discussed later. At $10 R_{J}$ the intensity values on the inbound passes are generally lower than those from the outbound segments. Additionally, the radial profiles of the outbound intensities are steeper than those of the inbound portions. This can be quantified by fitting an $R^{\delta}$ curve to the 11-20 $R J$ region; the resulting indices, derived with the standard least squares method, are given in Table 1. While the radial dependences in this region are adequately represented by power laws, nearly all orbits exhibit a flattening of the intensities beyond $20 R$.

Although there are differences in the local times of the inbound segments, the data do not seem to be better organized by local time. Recent work by Khurana [2001] has shown the magnetic field in the middle magnetosphere of Jupiter has a dependence on local time. Since the magnetic field governs much of the ions' motion, it is reasonable to expect $|B|$ to better organize the HIC oxygen intensities. The average magnetic field magnitude $(<B>)$ was obtained for each selected interval and is 
Table 1. Resulting Parameters From Fits of $R^{\S},\left\langle B>^{\alpha}\right.$ and $E^{\natural}$ to Oxygen Intensity Between 11 and $20 R_{J}$

\begin{tabular}{|c|c|c|c|c|c|c|c|c|}
\hline \multirow[b]{2}{*}{ Orbit } & \multicolumn{4}{|c|}{ Inbound } & \multicolumn{4}{|c|}{ Outbound } \\
\hline & $\delta$ & $\alpha$ & $\bar{\beta}$ & $\beta+1$ & $\delta$ & $\alpha$ & $\bar{\beta}$ & $\beta+1$ \\
\hline E4 & $9.1 \pm 1.1$ & $2.9 \pm 0.5$ & $2.7 \pm 0.1$ & $3.7 \pm 0.1$ & $15.8 \pm 1.9$ & $4.3 \pm 0.9$ & $2.8 \pm 0.2$ & $3.8 \pm 0.2$ \\
\hline E6 & $9.5 \pm 0.9$ & $\ldots$ & $2.9 \pm 0.1$ & $3.9 \pm 0.1$ & $14.9 \pm 2.2$ & $-\sim^{a}$ & $2.7 \pm 0.1$ & $3.7 \pm 0.1$ \\
\hline G7 & $14.4 \pm 1.0$ & $4.2 \pm 0.5$ & $2.8 \pm 0.1$ & $3.8 \pm 0.1$ & $17.0 \pm 1.3$ & $4.8 \pm 0.6$ & $2.7 \pm 0.1$ & $3.7 \pm 0.1$ \\
\hline G8 & $12.5 \pm 1.1$ & $3.8 \pm 0.6$ & $2.7 \pm 0.1$ & $3.7 \pm 0.1$ & $16.8 \pm 1.3$ & $5.7 \pm 0.8$ & $3.1 \pm 0.1$ & $4.1 \pm 0.1$ \\
\hline C9 & $9.8 \pm 0.7$ & $3.2 \pm 0.5$ & $3.0 \pm 0.1$ & $4.0 \pm 0.1$ & $16.8 \pm 1.4$ & $4.1 \pm 0.5$ & $3.1 \pm 0.1$ & $4.1 \pm 0.1$ \\
\hline $\mathrm{C} 10$ & $10.7 \pm 1.1$ & $3.0 \pm 0.4$ & $2.7 \pm 0.1$ & $3.7 \pm 0.1$ & $10.3 \pm 1.2$ & $3.4 \pm 0.5$ & $2.8 \pm 0.1$ & $3.8 \pm 0.1$ \\
\hline E11 & $14.3 \pm 2.9$ & $7.7 \pm 3.5$ & $2.7 \pm 0.1$ & $3.7 \pm 0.1$ & $16.4 \pm 1.5$ & $4.9 \pm 0.4$ & $3.3 \pm 0.2$ & $4.3 \pm 0.2$ \\
\hline $\mathrm{C} 21$ & $9.3 \pm 1.5$ & $2.8 \pm 0.7$ & $3.0 \pm 0.1$ & $4.0 \pm 0.1$ & $11.4 \pm 1.1$ & $4.5 \pm 0.6$ & $3.0 \pm 0.2$ & $4.0 \pm 0.2$ \\
\hline $\mathrm{C} 23$ & ...b & $\ldots$ & $\ldots$ & $\ldots$ & $12.7 \pm 1.1$ & $4.4 \pm 0.3$ & $2.7 \pm 0.2$ & $3.7 \pm 0.2$ \\
\hline
\end{tabular}

${ }^{2}$ No magnetic field data available for this orbit.

${ }^{b}$ Not enough points in the 11-20 $R$, region for a fit.

used in the bottom panels of Plate 1 . However, the orbit-to-orbit variation is not reduced in either the inbound and outbound segments when plotted versus $\langle B>$, suggesting the orbit-to-orbit variation is related to temporal variations. This is further supported by the substantial differences between inbound orbits $\mathrm{C} 21$ and $\mathrm{C} 23$. Both were obtained at local times significantly different (closer to midnight) than the other orbits, yet $\mathrm{C} 23$ agrees well with the $\langle B>$ organization seen in $\mathrm{E} 4, \mathrm{C}$, and $\mathrm{ClO}$ (and the inner part of G7).

During the outbound E6 pass, there is a significant increase in the oxygen intensity near $20 R J$ which is similar to events discussed by Selesnick et al. [2001]. The authors found occurrences of plasma sheet thinning in association with such events and concluded that they were evidence of acceleration occurring in the 25-30 $R_{J}$ region. Similar events were observed in the EPD data and discussed by Woch et al. [1998]. The newly accelerated material diffuses inward and has been observed in the inner magnetosphere by energetic particle detectors on the Voyager, Pioneer, and Galileo spacecraft [Vogt et al., 1979a, 1979b; Gehrels et al., 1981; Gehrels and Stone, 1983; Garrard et al., 1996; Cohen et al., 2000]. That the increase in E6 occurs significantly inside of $25 R J$ is consistent with the inward diffusion indicated by the simple diffusion model presented later. Additional acceleration signatures are evident in the E4 and C9 inbound segments as well.

For each of the magnetic equator crossings, the LET B oxygen data were divided into four energy intervals to create a spectrum. The spectra obtained for different radial positions for the $\mathrm{C10}$, $\mathrm{E} 4$, and $\mathrm{C} 9$ inbound and $\mathrm{G} 7$ outbound passes are plotted in Figure 4. Although sufficient statistics to obtain all four spectral points for every crossing are not always available, it is clear in $\mathrm{C10}, \mathrm{C9}$, and G7 that the spectra are statistically consistent with power laws where the spectral indices are independent of radial distance (average $\chi^{2}$ values are 1.1 and 1.6 for the inbound and outbound orbits, respectively). This is true for nearly all the orbits both inbound and outbound and spectral indices $(\beta)$ near -2.8 are typical (Table 1).

For several orbits there are spectra such as that obtained at 21 $R_{J}$ in E4. These spectra typically have a peak near $7 \mathrm{MeV}$ nucleon ${ }^{-1}$ which persists for several $R_{\mathrm{J}}$. At distances both inside and outside this region the spectra are well-behaved power laws. These peaked spectra may be further evidence of acceleration of plasma to high energies and predominantly occur in the 20-30 $R_{J}$ region.

The observed radial intensity variations can be compared with expectations based on radial diffusion theory. Assuming that there are no significant losses or sources within, or in close proximity to, the region of interest, then the ion phase space density should have no radial dependence. For a given magnetic moment $(M), L$ shell $(L)$, and $90^{\circ}$ pitch angle particles, the phase space density $(N)$ can be expressed in the following manner [Gehrels and Stone, 1983]:

$$
f\left(M, L, 90^{\circ}\right)=\frac{\gamma(M, L)}{2 m M^{2}} \frac{J_{\perp}(M, L)}{B^{2}},
$$

where $\gamma$ is the spectral index of integral intensity at the equator $\left(J_{D}\right), \mathrm{m}$ is the particle mass and $B$ is the magnetic field magnitude. For $f$ to be constant, $J_{\perp} \sim B^{2}$ for a given $M$. Thus the integral intensity can be expressed as

$$
J_{\perp}(M, L)=k B^{2} M^{-\gamma}
$$

or using $E=M B$,

$$
J_{\perp}(E=M B, L)=k B^{2}(E / B)^{-\gamma} \sim B^{\gamma+2} .
$$

If the $B$ dependence of the intensity is expressed as a power law with an index $\alpha$ and the energy spectrum of the differential intensity is a power law with index $-\beta$, then for locally lossless diffusion $\alpha=\beta+1$ (since $\beta=\gamma+1)$.

Using the $\beta$ variables resulting from the fits to the energy spectra, the expected $\alpha$ s $(=\beta+1)$ for each orbit segment assuming a constant phase space density is compared in Table 1 to the $\alpha$ values obtained by fitting the $\langle B>$ dependence of the intensities. A graphical comparison of the data and the expected dependence is presented in Plate 2. For most of the orbits, locally lossless diffusion is a good approximation. This is not unexpected since at these distances the pitch angle loss cone is very small, $\sim 0.4^{\circ}$. Thus even at the strong pitch angle scattering limit, very few particles are being lost. This assumes that radial diffusion is fast enough that strong losses near Io do not significantly influence the ion phase space densities at $R>10 R_{J}$. Such a case is illustrated in the radial diffusion model described below.

The statistics for carbon and sulfur are significantly less than those for oxygen. In order to investigate the radial dependence of the heavy ion composition, the equatorial crossings for all eight orbits, for which there is magnetic field data available, have been combined. The data were binned in radial distance and $\mathrm{O} / \mathrm{C}$ and $\mathrm{S} / \mathrm{C}$ ratios calculated. These resulting values as a function of $R_{J}$ are given in Figure 5. Typical solar values are indicated for reference [Reames, 1995] and mean magnetic moment values 

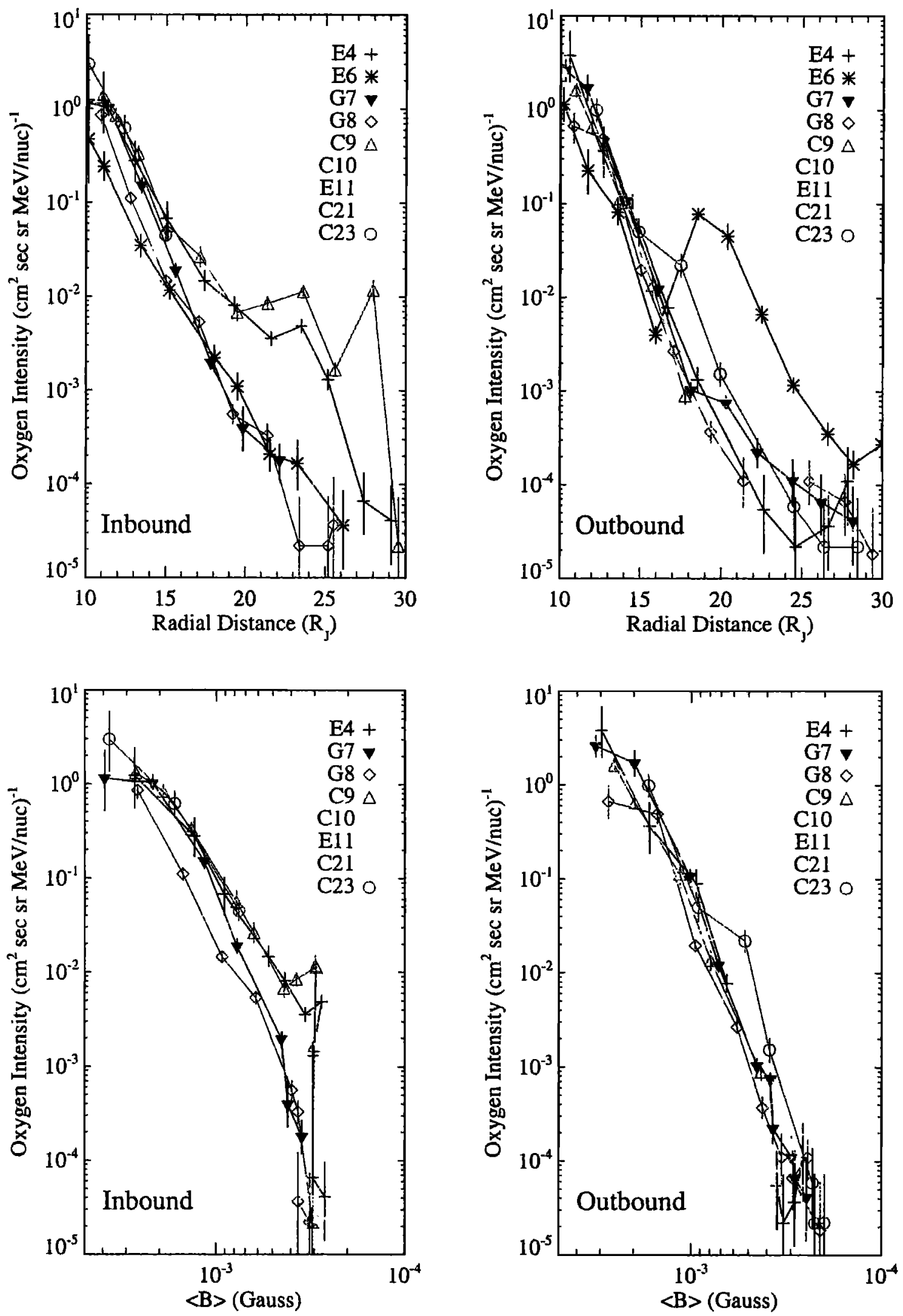

Plate 1. Oxygen intensities ( 4-18 MeV nucleon $\left.{ }^{-1}\right)$ for the inbound and outbound segments of each orbit (top) as a function of radial distance from Jupiter and (bottom) as a function of the average magnetic field magnitude. Note orbit E6 data are not presented in the bottom panels due to lack of magnetometer data for this orbit. 

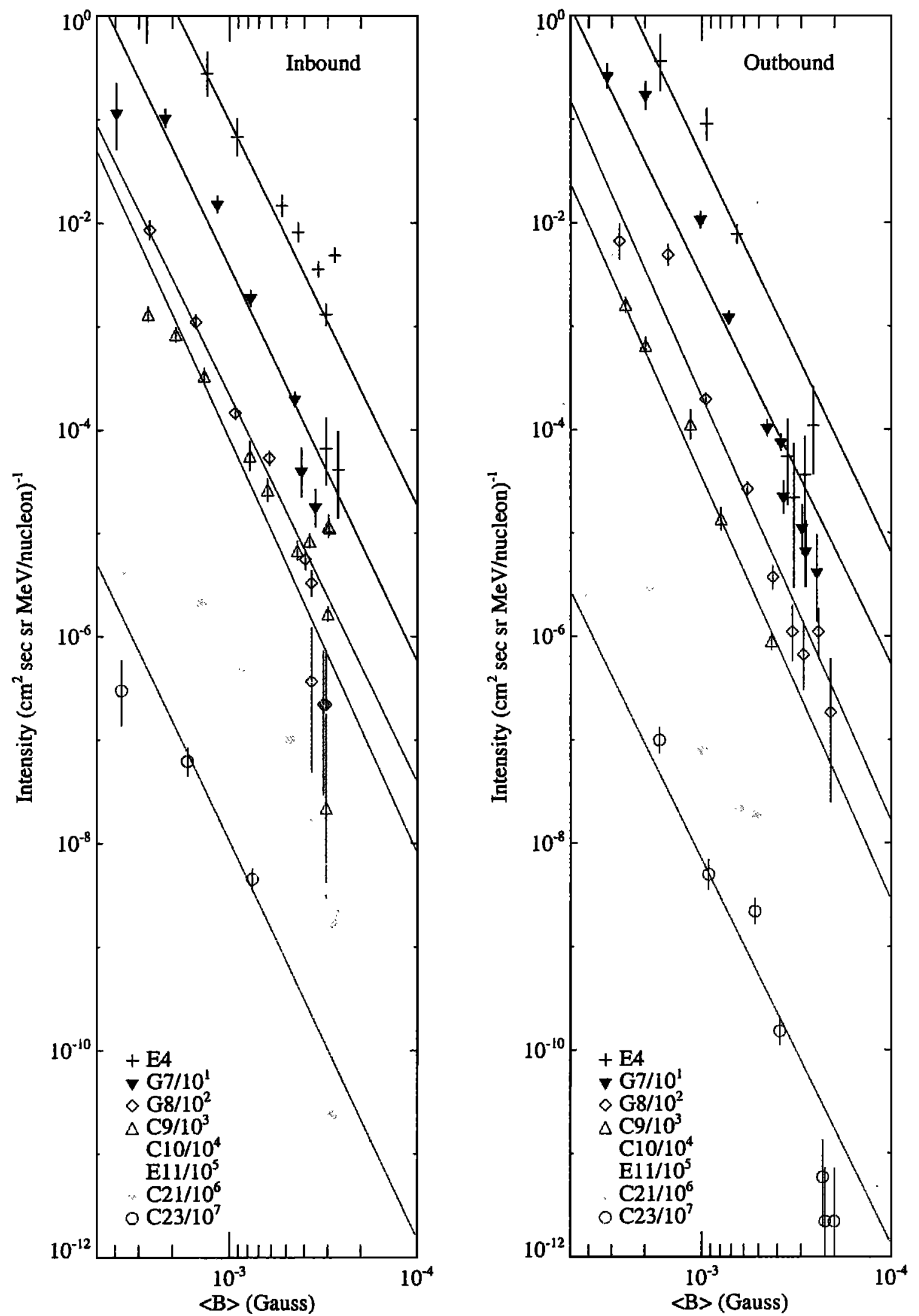

Plate 2. Oxygen intensities for the region $10-30 R_{J}$ for each orbit, inbound and outbound as a function of average magnetic field magnitude. The lines are the expected dependencies assuming lossless diffusion. Note that each orbit is shifted for clarity. 

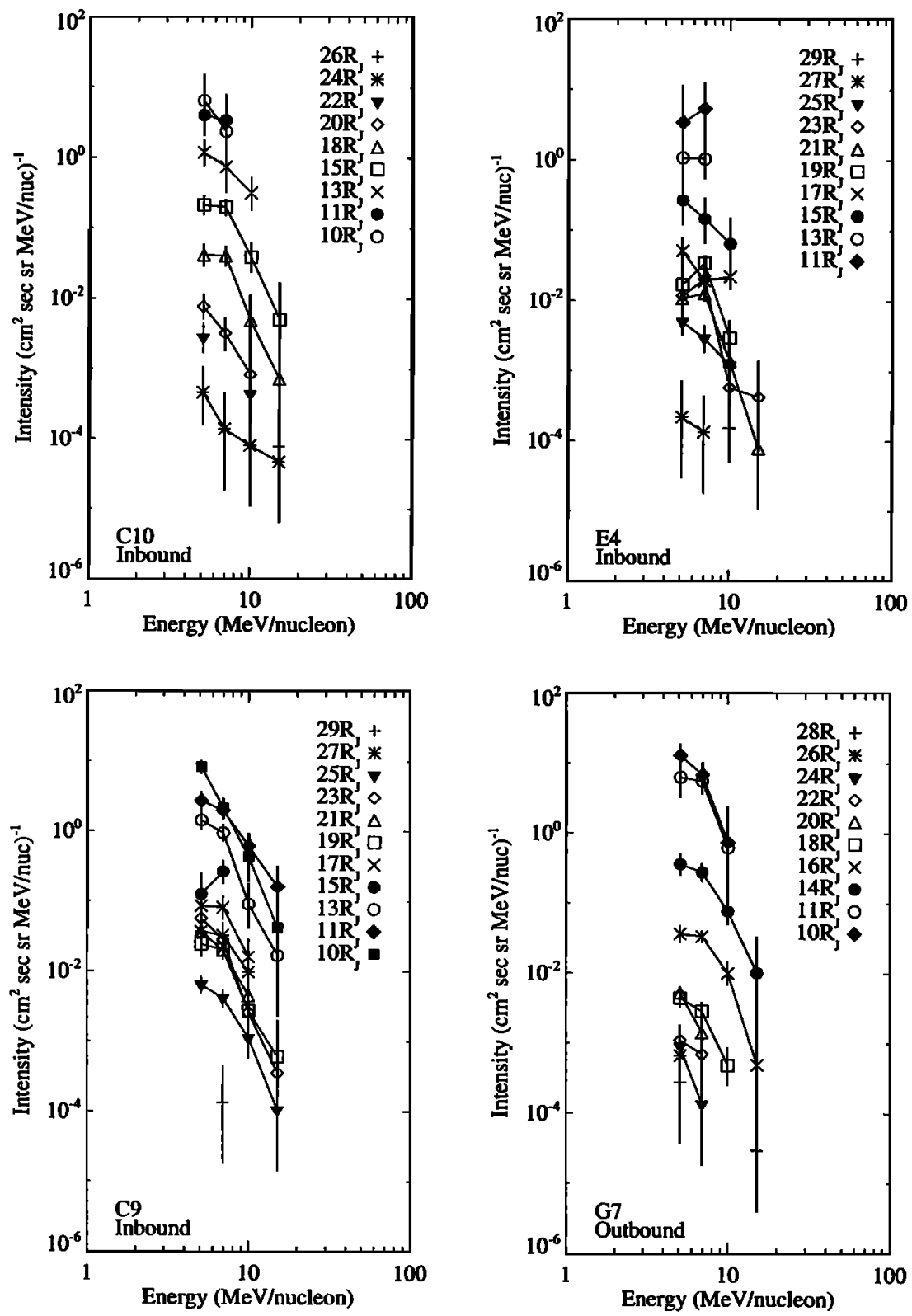

Figure 4. Oxygen energy spectra for the inbound portions of orbits C10, E4, and C9 and the outbound portion of orbit G7 taken at different radial distances from Jupiter.

(calculated from the observed dependence of $|B|$ on $R_{J}$ ) are given on the top $x$ axis.

Beyond 15-20 $R_{J}$ the $\mathrm{O} / \mathrm{C}$ and $\mathrm{S} / \mathrm{C}$ ratios are similar to that of solar material. With decreasing distance, the composition changes with $\mathrm{O} / \mathrm{C}$ and $\mathrm{S} / \mathrm{C}$ increasing. Since both $\mathrm{O}$ and $\mathrm{S}$ have Iogenic origins (as well as solar) and $C$ does not, this indicates a shifting from the dominance of solar material to a dominance of Iogenic material. In the inner magnetosphere, $\mathrm{O} / \mathrm{C}=38.5 \pm 10.4$ and $\mathrm{S} / \mathrm{C}=46.2 \pm 7.7$ values were measured by $\mathrm{HIC}$ (derived from reported $\mathrm{C} / \mathrm{O}$ and $\mathrm{S} / \mathrm{O}$ ratios [Garrard et al., 1996]), consistent with the trend seen in Figure 5.

\section{Interpretation and Model}

Observations of $\mathrm{C} / \mathrm{O}$ and $\mathrm{S} / \mathrm{O}$ abundance ratios made at lower energies in the 10-30 $R_{J}$ region are quite different with the values presented here. Values of 10 at $10 R_{J}, 5$ at $30 R_{J}$ for $\mathrm{O} / \mathrm{C}$ and 6 at
$10 R_{J}, 2$ at $30 R_{J}$ for $\mathrm{S} / \mathrm{C}$ (derived from reported $\mathrm{C} / \mathrm{O}$ and $\mathrm{S} / \mathrm{O}$ values) were obtained from the Low-Energy Charged Particle (LECP) instruments on Voyager 1 and 2 by Hamilton et al. [1981]. These values suggest a dominance of Iogenic material throughout the middle magnetosphere at energies of 0.60 to 1.15 $\mathrm{MeV}$ nucleon ${ }^{-1}$. Similar observations were made by the Energetic Particles Detector (EPD) experiment on Galileo [Kane et al., 1999] at 0.04 to $0.4 \mathrm{MeV}$ nucleon $^{-1}$ and the Heliosphere Instrument for Spectra, Composition, and Anisotropy at Low Energies (HI-SCALE) on Ulysses at 0.6 to $6 \mathrm{MeV}$ nucleon $^{-1}$ [Lanzerotti et al., 1992].

This apparent inconsistency can be understood in light of a suggestion by Hamilion et al. [1981]. The authors found that a common spectral shape adequately described the spectra for all observed heavy ion species (independent of solar or Iogenic origin) when the differential intensities were plotted as a function of energy per charge $(E / Q)$ as opposed to energy per nucleon 


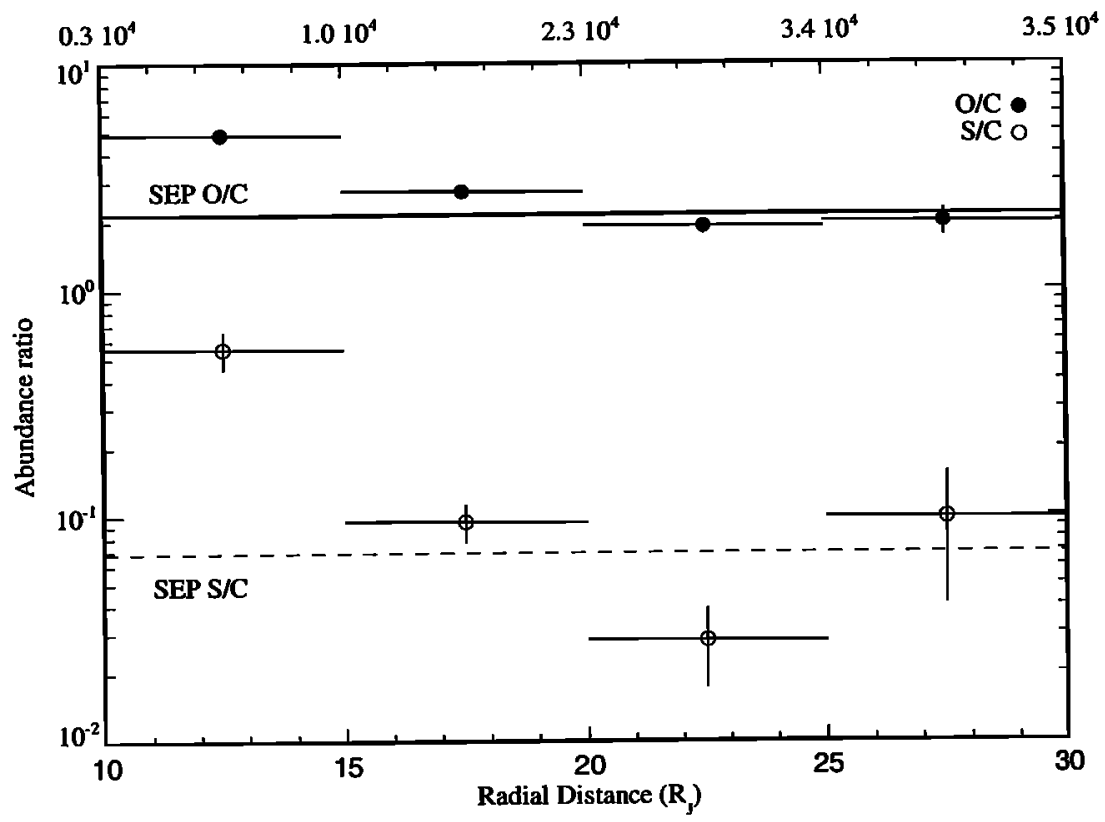

Figure 5. Oxygen to carbon and sulfur to carbon abundance ratios as a function of radial distance for all orbits combined. The horizontal lines are drawn at the solar particle values. Top scale is approximate corresponding magnetic moments sampled by HIC.

$(E / m)$. In this representation the abundance ratios were independent of $E / Q$.

In a simple exercise we assume that the energetic $\mathrm{C}, \mathrm{O}$, and $\mathrm{S}$ ions can be represented by a common spectrum that is a power law of index -3 at $E / Q<60 \mathrm{MeV} \mathrm{el}^{-1}$ which smoothly changes to a power law of index -9 at $E / Q>60 \mathrm{MeV} \mathrm{el}^{-1}$. In converting the spectra to a function of $E / m$ we assume $C$ has a single solar component with a charge state of 6 . Oxygen and sulfur have two components representing the Iogenic and solar origins. The Iogenic charge states were taken to be 1 and the solar charge states taken as 14 and 8 for $S$ and $O$, respectively. Since an ion conserves its magnetic moment $(M)$, it is useful to convert the $E / m$ spectra into $M$ spectra. This is done using the magnetic field magnitude at $25 R_{J}$ in Figure 6. The top panel illustrates, for oxygen, the $M$ dependence of solar and Iogenic components as well as the resulting sum. The bottom panel shows the resulting spectra for carbon, oxygen, and sulfur. Also shown in the figure are representative ranges of $M$ that would be sampled in the 10$30 R_{J}$ region by the fixed energy range detectors of EPD, LECP, and HIC (using a mean energy for each detector and the $|B|$ versus $R_{J}$ curve observed by Galileo). Since $|B|$ increases with decreasing radial distance, a fixed energy detector will sample ions with decreasing $M$ as the spacecraft moves toward Jupiter.

It is apparent from Figure 6 that the relative abundance of $\mathrm{C}$, $\mathrm{O}$, and $\mathrm{S}$ observed by $\mathrm{HIC}$ at higher $M$ values would be substantially different from that observed by LECP and EPD at lower $\mathrm{M}$ values in this scenario. While moving from 10 to $30 R_{h}$ HIC would see a transition from significant amounts of Iogenic material to significant amounts of solar material (as represented by $S$ and $C$, respectively, in this exercise). The LECP and EPD instruments would not see such a transition and would observe composition that is dominantly Iogenic.

Although this scenario is a simple one, it is a possible explanation the strongly energy and radially dependent heavy ion composition. If such a common spectrum is a result of an acceleration mechanism that operates near $25 R$, the question remains whether such a source could populate the inner magnetosphere with energetic particles on reasonable timescales and produce the temporal, radial, and spectra characteristics seen in the HIC data. To investigate this, a simple diffusion model with an ion source such as that just described was created.

Diffusion of equatorial ions at constant first adiabatic invariant is described by

$$
\frac{d f}{d t}=L^{2} \frac{d}{d L}\left(\frac{D_{L L}}{L^{2}} \frac{d f}{d L}\right)-\frac{f}{\tau}+S,
$$

where $f$ is the phase space density, $D_{L L}$ is the radial diffusion coefficient, $\tau$ is a lifetime for local losses, and $S$ is the source rate. For $D_{L L}$ and $\tau$ the model of Gehrels and Stone [1983], developed to describe the Voyager Cosmic Ray Subsystem (CRS) data, is used. The authors gave a range of values consistent with those data and other constraints in which the upper and lower limits of both $D_{L L}$ and $\tau$ differed by a factor of 5.5. We use the geometric mean of these limits which gives $D_{L L}=10^{-2} \mathrm{~L}^{1.6} \mathrm{~d}^{-1}$ for $L>9, D_{L L}$ $=5.3 \times 10^{-5} \mathrm{~L}^{4} \mathrm{~d}^{-1}$ for $L<9$ (we ignore variations in $D_{L L}$ at $L<8$ ), and $\tau=1.4 \times 10^{-4} M^{1 / 2} L^{11 / 2}(4-3 / L)^{1 / 2}$ day, where the units of $M$ are $\mathrm{MeV}$ nucleon- $\mathrm{G}^{-1}$. The time unit is days but can be varied by a factor of $5.5^{1 / 2}=2.3$ in either direction to represent the upper and lower limits on the diffusion parameters.

A function of energy per charge $(E / Q)$ that is common to all ionic species, as discussed above, is used for the source $S$ at $L=$ 25 , and we assume the solar $C, O$, and $S$ have charge states of 6 , 8 , and 14 respectively, while Iogenic $O$ and $S$ each have charge state of 1 . The solar $C$ and $O$ have equal relative source abundances, as do the Iogenic $O$ and $S$. The solar $S / O$ source ratio is $\mathbf{0 . 1 4}$. The Iogenic to solar abundance ratio is 200 , which leads to Iogenic ions predominant at low $L$ and the solar ions predominant at high $L$, as observed.

The model boundary conditions are $f=0$ at $L=6$, to represent strong losses near the Io torus [Cohen et al., 2000], and $f=0$ at $L=30$, to represent rapid radial transport at higher $L$ values [Selesnick et al., 2001]. The initial condition is $f=0$ for all $L$. 

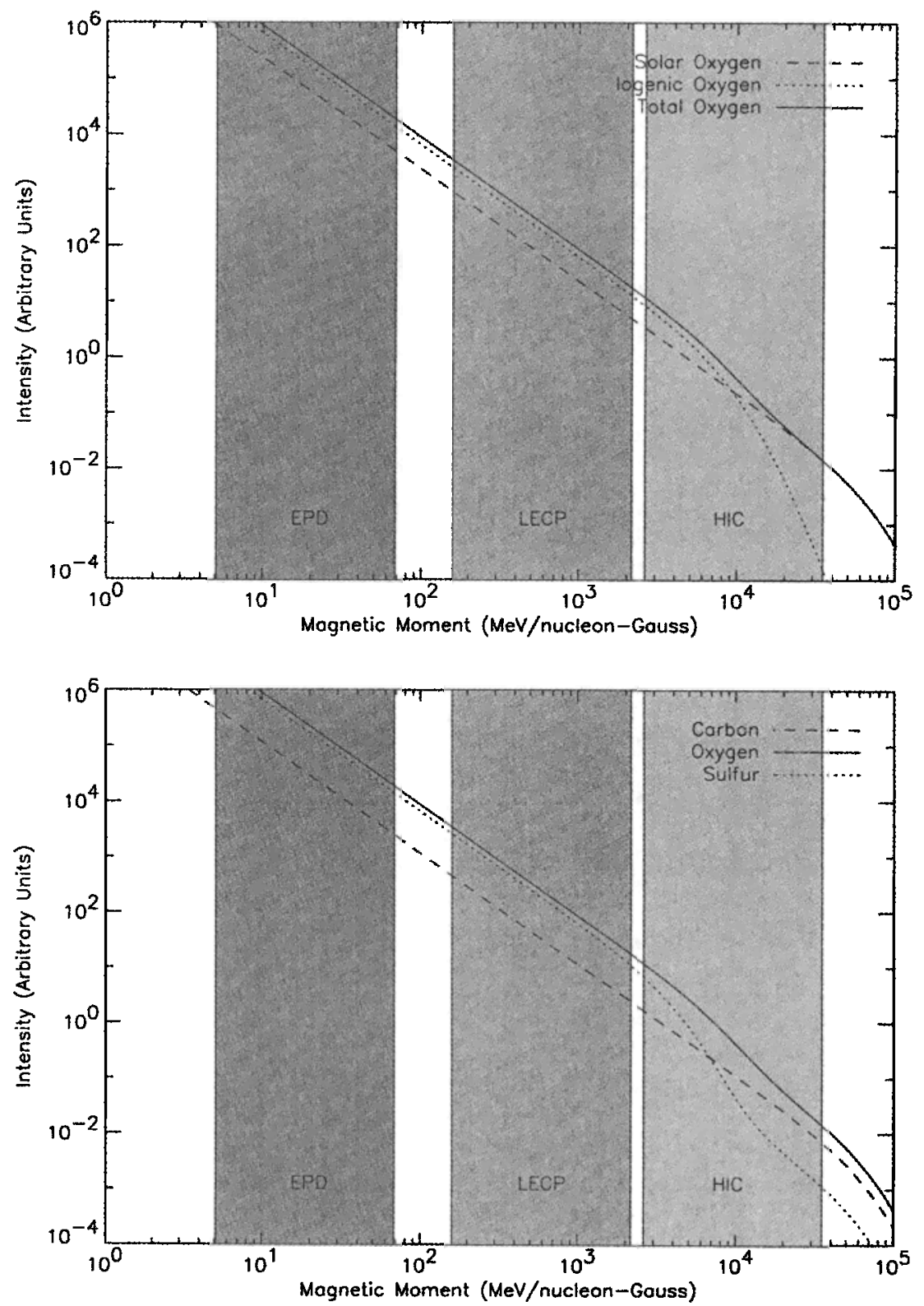

Figure 6. (a) The resulting spectrum of oxygen when a common energy per charge spectrum is converted to magnetic moment $(M)$ using assumed charge states of 1 for the Iogenic component and $\mathbf{8}$ for the solar. (b) The resulting spectra for oxygen, sulfur and carbon, using charge states of 6 for carbon (solar only) and 1 and 14 for sulfur Iogenic and solar components, respectively. oxygen and solar oxygen respectively. Shaded areas indicate the $M$ interval sampled by HIC, LECP, and EPD from 10 to $30 R_{\text {s. }}$

We use a dipole magnetic field to convert the magnetic moment to energy. The source is active for the first 5 days of the simulation and then reduced to $S=0$. The model equation (4) is solved numerically for a simulated period of 50 days and $f$ is then converted to intensity $j=p^{2} f$ as a function of energy per nucleon. The results are shown in Figure 7.

The energy per nucleon spectra at $L=25$ and $t=5$ days (Figure 7a) show the effect of converting the source spectra from energy per charge. The $O$ spectrum includes solar and Iogenic contributions, so it is similar to the sum of the $\mathrm{C}$ and $\mathrm{S}$ spectra. The $O$ intensities as a function of $L$ at 5-day intervals (Figure $7 \mathrm{~b}$ ) have been integrated over the LET $B$ energy range of 4.5 to 18.4 $\mathrm{MeV}$ nucleon ${ }^{-1}$ and divided by the difference of those limits for comparison with the data in Plate 1. They show that the source reaches low $L$ values after $\sim 20$ days, that a steep $L$ dependence at high $L$ values is obtained only a few days after the source is turned off, and that after reaching its maximum value the intensity decays relatively slowly, particularly at low $L$. The energy per nucleon spectra after 50 days and at $L$ intervals of 4 (Figure 7c) are relatively similar in the $10<L<30$ range (as is seen in the data in Figure 4) and soften considerably at $L<10$, because of strong losses relative to the diffusion rate in that region in addition to the source spectral shape, as observed [Gehrels and Stone, 1983; Cohen et al., 2000]. The intensities as a function of $L$ at $t=50$ days (Figure 7d) show the relative abundances of the solar and Iogenic ions in the LET B energy range at the end of the simulation. Here it is apparent that the solar material dominates beyond $L \sim 15$ at these energies, 

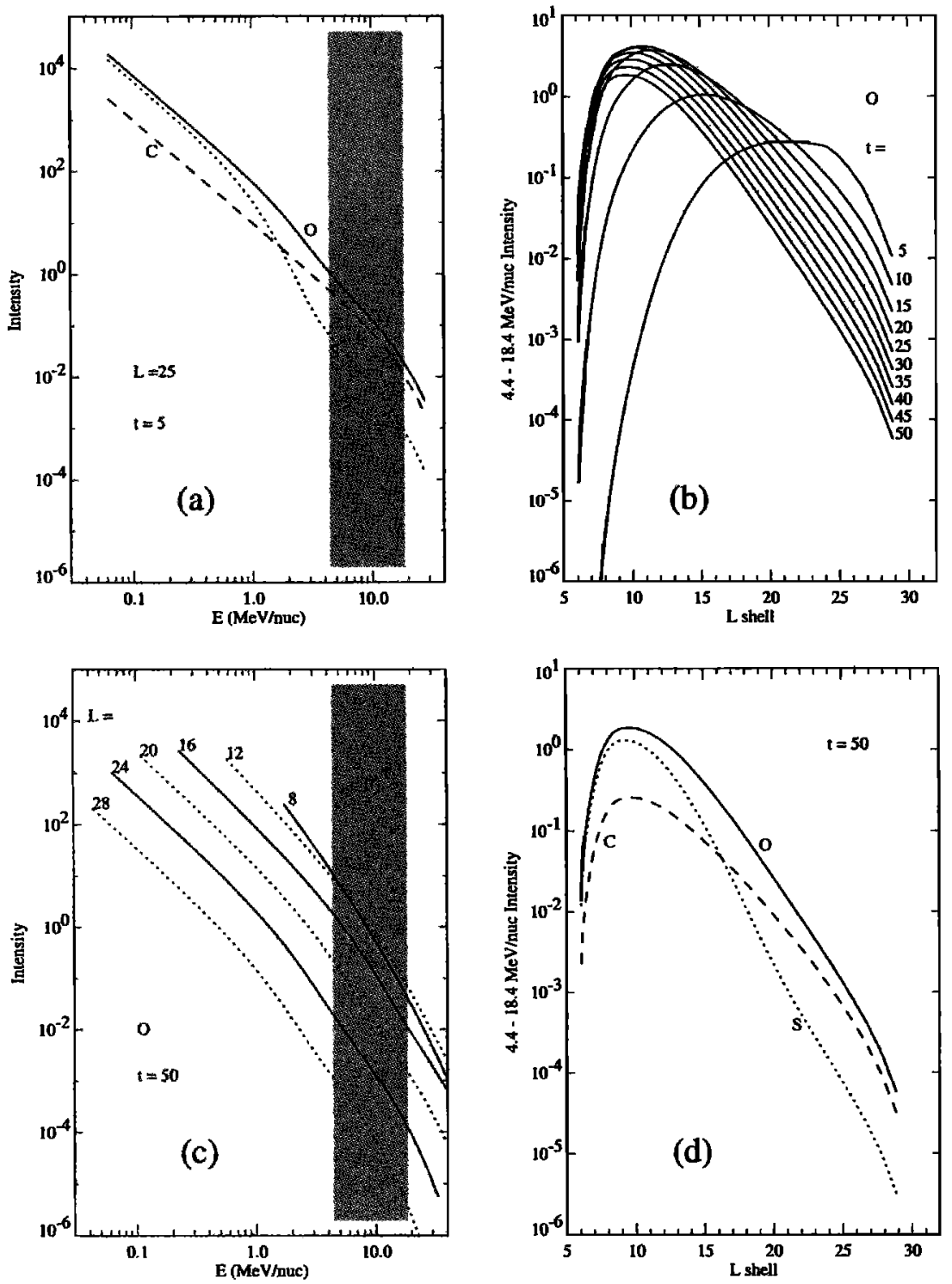

Figure 7. Resulting intensities as a function of (b and d) $L$, (c) energy, and (a) time (in days) for a diffusion model with a source spectnum which is a combination of solar and Iogenic material. Shaded areas indicate the energy interval of the HIC data.

consistent with the previously discussed observations. We note that since the model parameters have not been optimized to fit the data and a realistic field model is not used, only a qualitative agreement with the data should be expected. That the model qualitatively reproduces many of the behaviors seen in the data suggests that a combination of solar $\mathrm{O}$ and $\mathrm{C}$ and Iogenic $\mathrm{O}$ and $\mathrm{S}$ with a common $E / Q$ spectrum is a plausible source of the energetic ions observed in the middle magnetosphere.

\section{Summary}

We have presented observations of energetic ions in the middle Jovian magnetosphere, a region which has not been previously well examined. The radial dependence of the intensities, spectra and composition of energetic particles, obtained with the Heavy Ion Counter (HIC) on Galileo, indicate that at energies of $\sim 4.5$ to $18.4 \mathrm{MeV}$ nucleon $^{-1}$ solar material dominates the 15 to $30 R_{J}$ region and diffuses without loss toward Jupiter. The fact that the composition near 10-15 $R_{J}$ becomes more Iogenic is understood in terms of a common energy per charge spectrum for all heavy ions. It is the very different charge to mass ratios of the ions of solar origin as compared to those of Iogenic origin that leads to a composition that is strongly dependent on magnetic moment and therefore radial distance in the HIC energy range. This suggestion also accounts for the dissimilar composition measured by instruments of differing energy ranges.

Near $25 R_{J}$ there is evidence of acceleration of ions to $\mathrm{MeV}$ energies. The location of this acceleration has been suggested previously but not directly observed before the Galileo mission. A simple diffusion model indicates that a combined source of accelerated solar and logenic ions at $L=25$ can diffuse inward and substantially populate the $L<10$ region on a timescale of tens of days. This model qualitatively reproduces both the spectral and radial characteristics of the HIC data.

A common acceleration region and energy per charge spectrum for the ions observed by both HIC and EPD on Galileo suggests that a single mechanism may be responsible for the 
acceleration of the cold plasma from Io to energies of several $\mathrm{MeV}$ nucleon ${ }^{-1}$, although it is likely that many acceleration cycles would be required. Such a mechanism would also accelerate the observed solar particles that enter the magnetosphere from outside. Therefore it is likely that their phase space density should decrease with increasing distance beyond the acceleration site. Future work with the HIC data may confirm this and provide additional information on the acceleration process.

Acknowledgments. We thank the magnetometer team for making their data available to us. This work was supported by NASA grant NAG58634 under the Jovian System Data Analysis Program.

Janet G. Luhmann thanks the referees for their assistance in evaluating this paper.

\section{References}

Barbosa, D. D., A. Eviatar, and G. L. Siscoe, On the acceleration of energetic ions in Jupiter's magnetosphere, J. Geophys. Res., 89, 3789$3800,1984$.

Cohen, C. M. S., T. L. Garrard, E. C. Stone, J. F. Cooper, N. Murphy, and N. Gehrels, Io encounters past and present: A heavy ion comparison, J. Geophys. Res., 105, 7775-7782, 2000.

Garrard, T. L., N. Gehrels, and E. C. Stone, The Galileo heavy element monitor, Space Sci. Rev., 60, 305-315, 1992.

Garrard, T. L., E. C. Stone, and N. Murphy, Effects of absorption by lo on composition of energetic heavy ions, Science, 274, 393-394, 1996.

Gehrels, N., and E. C. Stone, Energetic oxygen and sulfur ions in the Jovian magnetosphere and their contribution to the auroral excitation, J. Geophys. Res., 88, 5537-5550, 1983.

Gehrels, N., E. C. Stone, and J. H. Trainor, Energetic oxygen and sulfur in the Jovian magnetosphere, J. Geophys. Res., 86, 8906-8918, 1981.

Hamilton, D. C., G. Gloeckler, S. M. Krimigis, and L. J. Lanzerotti, Composition of nonthermal ions in the Jovian magnetosphere, $J$. Geophys. Res., 86, 8301-8318, 1981.

Kane, M., et al., Galileo Energetic Particles Detector measurements of hot ions in the neutral sheet region of Jupiter's magnetodisk, Geophys. Res. Lett., 26, 5-8, 1999.

Khurana, $\mathrm{K}$., Influence of solar wind on Jupiter's magnetosphere deduced from currents in the equatorial plane, J. Geophys. Res., in press, 2001.
Krimigis, S. M., and E. C. Roelof, Low-energy particle population, in Physics of the Jovian Magnetosphere, edited by A. J. Dessler, p. 106, Cambridge Univ. Press, New York, 1983.

Lanzerotti, L. J., et al., The hot plasma environment at Jupiter: Ulysses results, Science, 237, 1518-1524, 1992.

Reames, D. V., Coronal abundances determined from energetic particles, Adv. Space Res., 15, (7)41-(7)51, 1995.

Schardt, A. W., and C. K. Goertz, High-energy particles, in Physics of the Jovian Magnetosphere, edited by A. J. Dessler, p. 157, Cambridge Univ. Press, New York, 1983.

Selesnick, R. S., C. M. S. Cohen, and K. K. Khuruna, Energetic ion dynamics in Jupiter's plasma sheet, $J$. Geophys. Res., J06, 18,895$18,905,2001$.

Simpson, J. A., et al., Energetic charged-particle phenomena in the Jovian magnetosphere: First results from the Ulysses COSPIN collaboration, Science, 257, 1543-1550, 1992.

Smith, E. J., K.-P. Wenzel, and D. E. Page, Ulysses at Jupiter: An overview of the encounter, Science, 257, 1503-1507, 1992.

Smith, E. J., and K.-P. Wenzel, Introduction to the Ulysses encounter with Jupiter, J. Geophys. Res., 98, 21,111-21,127, 1993.

Trainor, J. H., B. J. Teegarden, D. E. Stilwell, F. B. McDonald, E. C. Roelof, and W. R. Webber, Energetic particle population in the Jovian magnetosphere: A preliminary note, Science, 183, 311-313, 1974.

Vogt, R. E., et al., Voyager 1: Energetic ions and electrons in the Jovian magnetosphere, Science, 204, 1003-1007, 1979a.

Vogt, R. E., A. C. Cummings, T. L. Garrard, N. Gehrels, E. C. Stone, J. H. Trainor, A. W. Schardt, T. F. Conlon, and F. B. McDonald, Voyager 2: Energetic ions and electrons in the Jovian magnetosphere, Science, 206, 984-987, $1979 \mathrm{~b}$.

Williams, D. J., R. W. McEntire, S. Jaskulek, and B. Wilken, The Galileo Energetic Particles Detector, Space Sci. Rev., 60, 385-412, 1992.

Woch, J., N. Krupp, A. Lagg, B. Wilken, S. Livi, and D. J. Williams, Quasi-periodic modulations of the Jovian magnetotail, Geophys. Res. Lett., 25, 1253-1256, 1998.

C. M. S. Cohen and E. C. Stone, George W. Downs Laboratory of Physics, California Institute of Technology, MC 220-47, Pasadena, CA 91125, USA. (cohen@srl.caltech.edu)

R. S. Selesnick, The Aerospace Corporation, P.O. Box 92957M2/259, Los Angeles, CA 90009-2957, USA.

(Received January 10, 2001; revised August 24, 2001;

accepted August 27, 2001.) 